\title{
Las huellas invisibles de la violencia simbólica en la población adulta mayor: estudio comunitario*
}

\author{
The invisible traces of symbolic violence in the elderly \\ population: a community study
}

As marcas invisíveis da violência simbólica na população idosa: estudo comunitário

\author{
Daisy Valdivieso** , Aldrin Espín***, \\ Juan Manuel Guzmán**** , Elizabeth Vargas L.*****
}

\begin{abstract}
RESUMEN
El artículo propone algunas reflexiones sobre la violencia simbólica inmersa en la calidad de vida de adultos mayores, en el abandono, soledad, pobreza, condición física y de salud, afectando el ejercicio de sus derechos y dignidad, evidenciados en la complejidad social, su interrelación con la comunidad, el acceso a servicios, vivienda, alimentos, lengua de origen y desempleo que permanece invisible y naturalizado en las prácticas diarias.
\end{abstract} Palabras clave: violencia simbólica, calidad de vida, derechos, dignidad e identidad cultural, adultos mayores

\footnotetext{
* Esta investigación fue realizada bajo los principios éticos y académicos de la Universidad Central del Ecuador- UCE, Dirección de Investigación, CIF, Proyecto Semilla, 2019- 2020.

Se agradece a la CIF (Comisión de Investigación Formativa) de la Universidad Central por el apoyo y financiamiento de la investigación. Se agradece también al GAD Parroquial de San Antonio de Ibarra. Directivos/as de Pucahuaico 2019-2020.

** Docente e investigadora de la Universidad Central del Ecuador, Facultad de Ciencias Sociales y Humana, Carrera de Trabajo Social. Se agradece la colaboración de la docente: Myrian Peñaherrera y Hugo Jiménez: Colaboradores externo de investigación. davaldiviesos@uce.edu.ec

*** Docente e investigador de la Universidad Central del Ecuador, Facultad de Ciencias Sociales y Humana, Carrera de Trabajo Social.

**** Docente e investigador de la Universidad Central del Ecuador, Facultad de Ciencias Sociales y Humana, Carrera de Trabajo Social

***** Docente e investigadora de la Universidad Central del Ecuador, Facultad de Ciencias Sociales y Humana, Carrera de Trabajo Social
} 
El estudio, realizado entre 2019 y 2020, analiza estas condiciones en dos momentos: el primero, a través de una encuesta sociocontextual de percepción sobre la violencia, y el segundo a través de historias de vida en grupos focales de adultos mayores que reflejan que la violencia simbólica permanece casi imperceptible en su calidad de vida. Por eso, el interés de socializar y visibilizar su realidad, para interpelar al Estado ecuatoriano sobre una política pública adecuada en temas de prevención, mecanismos de protección, promoción de los derechos humanos y garantías efectivas en favor del adulto mayor, que permitan recuperar sus voces, su memoria e identidad como parte del compromiso y de la corresponsabilidad social.

Los resultados señalan la presencia de enfermedades crónicas que son más frecuentes en la vejez, el abandono familiar, el desánimo, la depresión y la soledad en la población de adultos mayores, así como la ausencia del Estado en la atención de la salud. Hay evidencia de discriminación basada en su etnia y en la edad, así como explotación laboral por actividades de cuidado no remuneradas, discriminación que en su mayoría proviene de su propio entorno familiar y de su comunidad. Por otra parte, los resultados reflejan un proceso de aculturación basado en la idea peyorativa de ser indígena, lo que se expresa en la presión social al interior de las familias para dejar atuendos, lenguaje e idiosincrasia proveniente de los pueblos originarios. Además, una corriente de recuperación de la identidad cultural desde los colectivos presentes y el gobierno local.

\section{SUMMARY}

The article proposes some reflections on the symbolic violence immersed in the quality of life of older adults. Abandonment, loneliness, poverty, physical and health conditions affect their rights and dignity, evidenced in the social complexity, interrelation with the community, access to services, housing, food, language of origin, and unemployment that remains invisible and naturalized in daily practices.

The study, conducted between 2019 and 2020, analyzes these conditions in two moments: the first, through a socio-contextual survey of perception of violence, and the second through life stories in focus groups of older adults that reflect that symbolic violence remains almost invisible in their quality of life. Therefore, our interest lies in socializing and making their reality visible. To question the Ecuadorian State about an adequate public policy on prevention, protection mechanisms, promo-
Key words: symbolic violence, quality of life, rights, dignity, and cultural identity, older adults 
tion of human rights, and adequate guarantees in favor of the elderly, to recover their voices, memory, and identity as part of the commitment and social co-responsibility. The results point to chronic diseases that are more frequent in old age, family abandonment, discouragement, depression, and loneliness in the elderly population, and the absence of the State in health care. There is discrimination based on their ethnicity and age and labor exploitation for unpaid care activities, discrimination that mostly comes from their family environment and community. On the other hand, the results reflect a process of acculturation based on the pejorative idea of being indigenous, which is expressed in the social pressure within families to leave behind the attire, language, and idiosyncrasy of native peoples. In addition, a current recovery of cultural identity from groups and local government.

\section{RESUMO}

O artigo propõe algumas reflexões sobre a violência simbólica imersa na qualidade de vida de idosos, no abandono, solidão, pobreza, condição física e de saúde, que afetam o exercício de seus direitos e dignidade, evidenciados na complexidade social, sua inter-relação com a comunidade, o acesso a serviços, moradia, alimentos, idioma de origem e desemprego que permanece invisível e naturalizado nas práticas diárias.

O estudo, realizado entre 2019 e 2020, analisa estas condições em dois momentos: o primeiro, através de uma pesquisa sociocontextual de percepção sobre a violência; o segundo, através de histórias de vida em grupos focais de idosos que refletem que a violência simbólica permanece quase imperceptível em sua qualidade de vida. Por isso, o interesse de socializar e visibilizar sua realidade para interpelar o Estado equatoriano sobre uma política pública adequada em temas de prevenção, mecanismos de proteção, promoção dos direitos humanos e garantias efetivas a favor do idoso, que permitam recuperar suas vozes, memória e identidade como parte do compromisso e da corresponsabilidade social.

Os resultados indicam a presença de doenças crônicas mais frequentes na velhice, o abandono familiar, o desânimo, a depressão e a solidão na população idosa, bem como a ausência do Estado na atenção à saúde. Existe evidência de discriminação em função da etnia e da idade, assim como de exploração laboral por atividades de cuidado não remuneradas, discriminação que, em sua maioria, é proveniente de seu próprio entorno familiar e

Palavras-chave: violência simbólica, qualidade de vida, direitos, dignidade e identidade cultural, idosos. 
de sua comunidade. Por outro lado, os resultados refletem um processo de aculturação baseado na ideia pejorativa de ser indígena, o que se expressa na pressão social dentro das famílias para abandonar vestimentas, linguagem e idiossincrasia proveniente dos povos originários. Ademais, uma corrente de recuperação da identidade cultural desde os coletivos presentes e do governo local. 


\section{Introducción}

La presente investigación analiza la calidad de vida y la percepción de la violencia de los adultos mayores de la comunidad de Pucahuaico, Parroquia San Antonio, Cantón Ibarra en el periodo 2019-2020. El Cantón Ibarra pertenece a la provincia de Imbabura y se ubica en la zona centro norte del Ecuador (imagen 1).

Histórica y políticamente, la población de este cantón inició un camino de aculturación en 1693, cuando fue nombrado como Corregimiento de Ibarra, lo cual indica una fuerte presencia colonial tanto en lo cultural como en lo administrativo; en 1861, ya en el periodo republicano, San Antonio se constituye como parroquia rural del naciente Ecuador con el nombre de San Antonio de los Caranquis (GADP San Antonio de Ibarra, 2015), nombre que es cambiado en 1870 por el actual San Antonio de Ibarra (Viteri, Villalva y César, 1988).

Estos antecedentes nos hacen ver, por un lado, la importante influencia del coloniaje en la zona y, por otro, la resistencia cultural al coloniaje. Ambas tendencias se visualizan en la autodenominación étnica, los usos y costumbres de la vestimenta, el lenguaje y las expresiones culturales que siguen luchando por no desaparecer.

Sobre la procedencia étnica de la población de San Antonio, investigadores como Herrera y Terán (2011), Morales (2017) y Viteri et al. (1988), reconocen sus orígenes precoloniales en tres asentamientos poblacionales: Huataviros, Tauriquies y Natabuelas, como parte de la confederación Caranqui, a quienes se les reconoce los mejores atributos de la cosmovisión andina. 


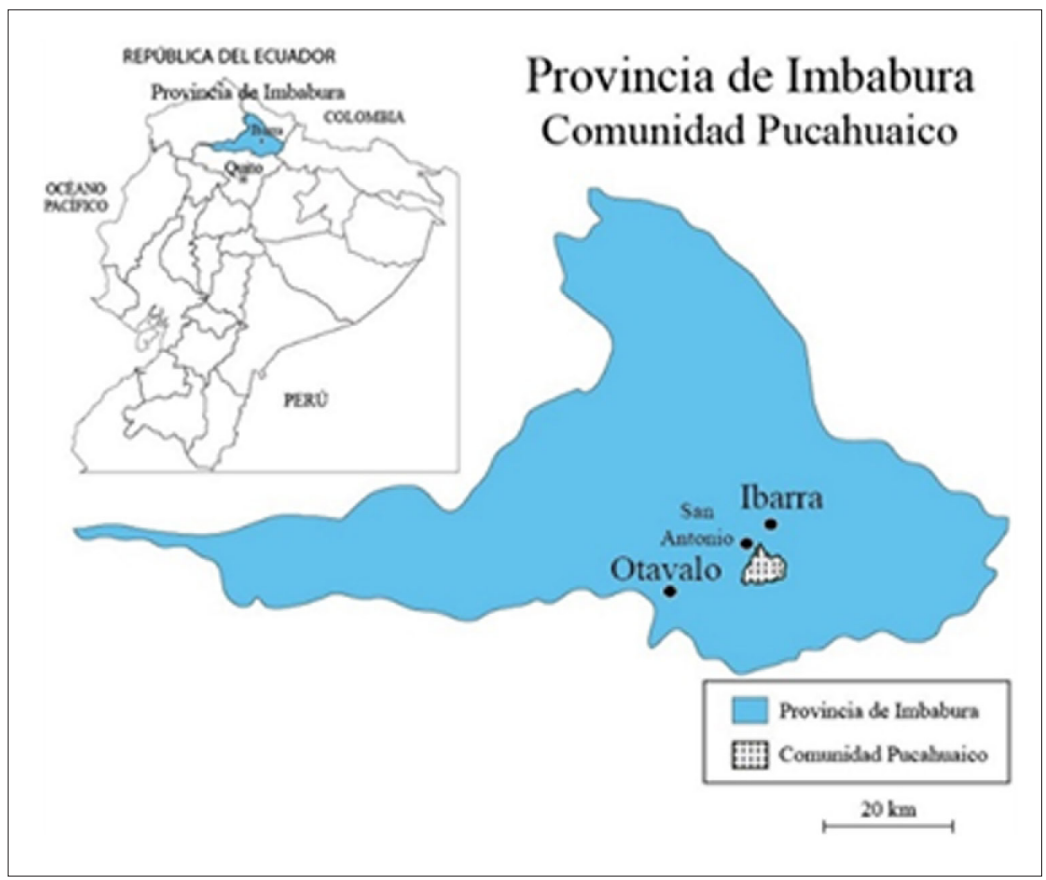

Imagen 1: Ubicación geográfica de la comunidad indígena Pucahuaico. Fuente: www.d-maps.com. Elaboración y adaptación equipo de investigación.

\section{Revisión teórica}

Para hablar de la violencia simbólica nos centramos en dos conceptos: la violencia simbólica desde la calidad de vida y la vulneración de sus derechos. En este sentido, esta revisión teórica permite analizar y explicar este fenómeno social en el ejercicio de derechos, entendiendo que la calidad de vida se refleja o está implícita en la vida cotidiana de los pueblos. Carballeda (2017, en Hermida y Meschini, 2018), para hablar de estas formas de violencia, expresa que se subsumen en la negación del otro, cuya respuesta es el sometimiento y las prácticas violentas.

Una violencia que Bourdieu (2008) identifica como eufemizada, que no es visible, es sutil y está presente en el trato y la reciprocidad a las personas. En esta misma línea, Fernández (2005), citando a Bourdieu (1999), expresa que esta violencia invisible tiene formas sutiles para instaurar su poder a través de prácticas que someten al otro. 
Bajo esta misma línea de razonamiento, y desde la especificidad del Trabajo Social, Carballeda (2017) incorpora la exclusión y desigualdad social como aspectos claves del discurso dominante que somete al individuo, vulnerando sus derechos como sujeto. Concordante con este pensamiento, Cazzaniga (2020) hace énfasis en que pensar al otro es reconocer al sujeto desde la intervención y no solo desde el discurso.

Por ello, en este pensamiento del otro Bourdieu y los trabajadores sociales mencionados enfatizan en que esta violencia se inscribe en la práctica diaria, y desde esta práctica debe repensarse la intervención. Agüero (2018) señala que, en esta práctica, además, guardan relación las significaciones de la vida social. Paralelamente, Ospina y Jiménez (2018) incorporan la categoría “vulnerabilidad social”, imbricada en la capacidad de autonomía económica social y el rol del Estado y la política pública como mecanismo decidor en la calidad de vida de estas familias. En concordancia con lo expresado por Ospina y Jiménez sobre vulnerabilidad social, Pizarro (2021) hace énfasis en que la misma debe estudiarse a partir de dos componentes explicativos: la inseguridad como reflejo de un suceso traumático y, por otro, la resiliencia como mecanismo de protección colectiva familiar y comunitaria.

Teorías, además, que se complementan con lo enunciado con Feito (2007) sobre la vulnerabilidad social desde la perspectiva de la bioética, en relación con el daño y las condiciones que intervienen en su calidad de vida que, en conjunto, conforman los componentes de la dignidad humana. Paralelamente, Gutiérrez (2013), al hablar de la dignidad humana, se inspira en la Corte Constitucional Colombiana (sentencia T-881 de 2002), en cuya sentencia se explicita que la misma incorpora elementos como la capacidad de vivir una vida libre de humillaciones, con autonomía, que signifique la no dependencia que genere relaciones de poder, que en conjunto serán características del vivir bien; es decir vivir como se quiera. Desde estos parámetros, Villa y Rivadeneira, L. (1999) destacan que las condiciones del envejecimiento generan cambios biológicos asociados a la edad, autopercepción sobre la importancia de su rol colectivo y familiar y el rol que le está siendo asignado en cada etapa cronológica del envejecimiento (en la Constitución ecuatoriana, un adulto mayor es aquel que ha cumplido 
los 65 años de edad, cuyo incremento es evidente en América Latina). Alvarado y Salazar (2014) estiman que, para 2030, los individuos de la generación llamada "baby boom" serán ancianos con enfermedades asociadas al proceso de envejecimiento.

\section{Estado del arte}

Siendo la violencia un problema de interés social merece una atención especial, ya que los problemas asociados a la violencia suelen estar ocultos y son complejos de visibilizar, principalmente en aquellas regiones en que la desigualdad económica, la pobreza y el desconocimiento de derechos profundizan el problema. En este contexto, revisaremos aquella bibliografía que, con base en estudios realizados en campo, han pretendido visibilizar la violencia, violencia que ha permanecido oculta en la sociedad y principalmente en los adultos mayores de pueblos indígenas.

La violencia puede estar oculta incluso hasta en las mismas instituciones de educación, violencias asociadas a discriminación, al género al pensamiento ideológico e incluso al lugar de procedencia. Miranda, Aguirre y Espín (2020) estudiaron las formas de violencia en una universidad de Quito, Ecuador, y concluyeron que no solo la violencia directa es la expresión más evidente: existen otras formas de violencia asociadas a aspectos culturales y simbólicos, incluyendo la discriminación como componente importante de la variable "violencia simbólica". El estudio determina que un $24 \%$ de la violencia corresponde a la violencia simbólica de discriminación. La violencia también fue objeto de estudio en instituciones de salud, en relación con el maltrato a adultos mayores en este tipo de instituciones. Pillemer y Moore (1990) concluyeron que el $81 \%$ del personal de enfermería había cometido maltrato psicológico, un $40 \%$ había abusado verbalmente, un $36 \%$ había sido testigo de maltrato físico y un $10 \%$ había cometido maltrato físico. Las formas de abuso en las instituciones son frecuentes. Sin embargo, no es fácil hacer visible este tipo de incidentes por parte de los funcionarios, personal médico o enfermería, por el temor a sanciones y tampoco los familiares denuncian por desconocimiento de derechos. Delgado y Enríquez (2010) estudiaron, en una comunidad indígena de Embera, Colombia, la violencia de género y entre generaciones mediante un enfoque de investigación de acción participativa. El estudio reveló un aumento en el maltrato 
entre hombres y mujeres; en consecuencia, ellas proponen revisar en qué medida la violencia intrafamiliar, así como la desvalorización de las relaciones entre generaciones y la pérdida de su propia cultura por la exposición a la cultura occidental, es causada por la violencia simbólica, lo que Galtung (1969) etiqueta como "violencia cultural" y Bourdieu (1991) como "violencia simbólica". Para Espín-León, Jimeno-Morenilla, Pertegal-Felices y Azorín-López (2021), como producto de la influencia de factores culturales occidentales, se estaría produciendo algún tipo de violencia en las comunidades indígenas, especialmente en los adultos mayores, a consecuencia de la migración de los indígenas jóvenes a ciudades con cultura occidental. Incluso, para el tratamiento médico contra la covid-19, la violencia estructural y el racismo institucional, expresados como violencia simbólica, inciden en la atención tardía a los pueblos indígenas. Cortez, Muñoz Martínez y Ponce-Jiménez (2020) mostraron que la letalidad es mayor en la población indígena que en la población en general, y concluyen que la vulneración estructural de los derechos y de equidad al acceso a la salud constituyen una forma de violencia.

La violencia es considerada por algunos autores y gobiernos como un problema de salud pública -inicialmente considerada como un problema de bienestar-. En este sentido, Gómez Ricárdez, Rodríguez Abrego y Krug Llamas (2007) realizaron un estudio a 1.078 adultos mayores de la comunidad indígena de Chiapas, México, sobre la prevalencia y los factores asociados a la violencia familiar. Mediante entrevistas domiciliarias recabaron información sobre presencia de enfermedades, estado cognitivo y violencia familiar, entre otras. Los resultados mostraron que la prevalencia de violencia en las familias indígenas de Chiapas es de un $8,1 \%$ y que los factores están fuertemente asociados al analfabetismo. Sin embargo, para Casanova Moreno, Trasancos Delgado, Corvea Collazo, Pérez Sierra y Prats Álvarez (2013) el problema de la violencia en el adulto mayor requiere, para su visualización, sensibilizar a los organismos o equipos de salud. Casanova, en un consultorio médico de la ciudad Pinar del Río, caracterizó las manifestaciones de violencia intrafamiliar en 86 adultos mayores diabéticos, en donde se determinó que el grupo de edad más maltratado fue las mujeres de 60 a 69 años, y que los agresores más cercanos fueron sus propios hijos. La investigación concluye que la comunidad no reconoce al maltrato de adultos mayores diabéticos como un problema de salud. 
Existen varios estudios sobre la violencia en el seno familiar. Rodríguez Calvo, Gómez Mendoza, Guevara de León, Arribas Llopis, Duarte Durán y Ruiz Álvarez (2018) caracterizaron la violencia intrafamiliar en el adulto mayor desde información de 314 pacientes de más de 60 años, con un muestreo no probabilístico mediante entrevistas no estructuradas en la provincia de Villa Clara. Los resultados mostraron que la mayor cantidad de maltrato es de tipo psicológico y físico; psicológico expresado en violencia simbólica a través de agresiones verbales o de gestos, con el afán de humillar, atemorizar, quitar la libertad y aislar al adulto mayor de toda convivencia social. Violencia que se produce en aquellos ancianos que viven junto a sus hijos y nietos, siendo más evidente en aquellos ancianos con sexo femenino. Otro estudio describe la prevalencia de la violencia doméstica y la brecha de género en adultos mayores de 65 a 74 años en las ciudades de Natal, Brasil, y Manizales, Colombia. Los resultados muestran que la violencia psicológica en las mujeres fue la más alta y, de esta violencia, la recibida por parte de su propio compañero tiene un $25,7 \%$ en la ciudad de Natal y un $19,4 \%$ en la ciudad de Manizales, seguida de la violencia causada por algún miembro de la familia, con 18,3\% en Manizales y un $10 \%$ en Natal. El estudio concluye que la prevalencia de violencia en adultos mayores debe ser considerada como prioridad en las políticas de salud en Latinoamérica (Guedes, Curcio, Alvarado Llano, Zunzunegui y Guerra 2015).

Para Francés (2005) la variable simbólica tiene un rol importante en todo este proceso, ya que los propios actores sujetos de violencia le otorgan legitimidad y consentimiento gracias a la perspicacia con la cual actúa.

\section{Apuntes metodológicos}

El estudio se realizó en la comunidad de Pucahuaico. Población finita, determinada a través de información otorgada por la Junta parroquial de San Antonio, en la que se detallan datos macros de las comunidades urbano-rurales y rurales de la Comunidad, y datos del último Censo de 2019-2020ㄹ, que levantó la directiva de Pucahuaico.

1 Ecuador, San Antonio de Ibarra, GAD Parroquial, Plan de Ordenamiento Territorial, (PDOT, 2014) y Censo Poblacional elaborado por la Directiva del Comité Barrial de Pucahuaico, periodo 2019- 2020. 
La investigación consistió en dos fases. En la primera se utilizó un método aleatorio simple, que consistió en agrupar a la población, mediante invitación a participar de una actividad grupal en la casa barrial de Pucahuaico, luego de lo cual se seleccionaron al azar los individuos que serían encuestados. La segunda fase consistió en encuestas a partir de las entrevistas, utilizando el método no probabilístico en el que se seleccionan parámetros bajo elementos que caracterizan vulnerabilidad en los adultos mayores.

La entrevista se aplicó a los 49 asistentes, hombres y mujeres en edades comprendidas entre 18 a 85 . La encuesta se hizo a través de la técnica de "bola de nieve" para acceder a un mayor número de población (Otzen y Manterola, 2017) y consistió en 34 ítems, siete de los cuales corresponden a preguntas generales y 27 a preguntas referentes al contexto social de los adultos mayores. El instrumento de la encuesta obedece a orientar el fenómeno social a través de un indicador que devele vulnerabilidad en los adultos mayores, y que esta sea concebida para los investigadores como violencia simbólica, Además, se trabajó en unidades de análisis que no se encuentran en un listado previamente definido (Muñoz Loayza, 2018) (ver tabla 1).

Luego de las entrevistas se identificó a 10 adultos mayores que tenían algún tipo de vulnerabilidad, y se aplicó una encuesta a profundidad, basada en cuatro categorías y 14 unidades semánticas que exploran, desde la mirada teórica integral (Ungar, 2008), la resiliencia en los diferentes niveles ecológicos, desde las características individuales, familiares y socioculturales, las mismas que orientaron a la identificación de la variable "violencia simbólica" en esta población (ver tabla 2). Estas categorías y unidades son relevantes en la articulación de redes de solidaridad entre pares identificadas en las entrevistas aplicadas. 
Tabla 1.

Ítems aplicados en la encuesta.

\begin{tabular}{|c|c|c|c|}
\hline \multicolumn{4}{|c|}{ Aplicación de los ítems } \\
\hline Items & 18-30 años & 31-64 años & $>65$ \\
\hline \multicolumn{4}{|l|}{ Ítems de tipo general } \\
\hline Nombre de la persona encuestada* & $\mathrm{x}$ & $\mathrm{x}$ & $\mathrm{x}$ \\
\hline Edad en la que se encuentra & $\mathrm{x}$ & $\mathrm{x}$ & $\mathrm{x}$ \\
\hline Género & $\mathrm{x}$ & $\mathrm{x}$ & $\mathrm{x}$ \\
\hline Nivel de educación & $\mathrm{x}$ & $\mathrm{x}$ & $\mathrm{x}$ \\
\hline Estado civil & $\mathrm{x}$ & $\mathrm{x}$ & $\mathrm{x}$ \\
\hline \multicolumn{4}{|l|}{ Ítems del contexto social } \\
\hline ¿A qué etnia pertenece? & $\mathrm{x}$ & $\mathrm{x}$ & $\mathrm{x}$ \\
\hline Número de hijos/as que tiene & $\mathrm{x}$ & $\mathrm{x}$ & $\mathrm{x}$ \\
\hline $\begin{array}{l}\text { ¿Las personas adultas mayores reciben } \\
\text { un pago por su trabajo? }\end{array}$ & $\mathrm{x}$ & $\mathrm{x}$ & \\
\hline $\begin{array}{l}\text { ¿Los adultos mayores que existen en } \\
\text { su comunidad necesitan ayuda para } \\
\text { movilizase? }\end{array}$ & $\mathrm{x}$ & $\mathrm{x}$ & \\
\hline $\begin{array}{l}\text { ¿Las personas adultas mayores reciben } \\
\text { respeto y cariño de sus familiares? }\end{array}$ & $\mathrm{x}$ & $\mathrm{x}$ & \\
\hline $\begin{array}{l}\text { ¿Las personas adultas mayores } \\
\text { participar en las actividades y } \\
\text { decisiones que se toman en su barrio o } \\
\text { comunidad? }\end{array}$ & $\mathrm{x}$ & $\mathrm{x}$ & \\
\hline $\begin{array}{l}\text { ¿Las personas adultas mayores se } \\
\text { sienten abandonadas/aisladas por su } \\
\text { familia? }\end{array}$ & $\mathrm{x}$ & $\mathrm{x}$ & \\
\hline $\begin{array}{l}\text { ¿Los familiares de las personas adultas } \\
\text { mayores se apropian de los bienes o } \\
\text { dinero sin su consentimiento? }\end{array}$ & $\mathrm{x}$ & $\mathrm{x}$ & \\
\hline $\begin{array}{l}\text { ¿La familia de los adultos mayores } \\
\text { se avergüenza de reconocer como } \\
\text { indígena a sus progenitores (padre/ } \\
\text { madre)? }\end{array}$ & $\mathrm{x}$ & $\mathrm{x}$ & \\
\hline $\begin{array}{l}\text { ¿Las familias de las personas adultas } \\
\text { mayores les delegan el cuidado de sus } \\
\text { nietos u otras responsabilidades? }\end{array}$ & $\mathrm{x}$ & $\mathrm{x}$ & \\
\hline $\begin{array}{l}\text { ¿Conozco las leyes que protegen a los } \\
\text { adultos mayores? }\end{array}$ & $\mathrm{x}$ & $\mathrm{x}$ & \\
\hline $\begin{array}{l}\text { ¿Existen servicios de atención médica e } \\
\text { integral gratuita para adultos mayores? }\end{array}$ & $\mathrm{x}$ & $\mathrm{x}$ & \\
\hline
\end{tabular}




\begin{tabular}{|c|c|c|}
\hline $\begin{array}{l}\text { ¿Las personas adultas mayores tienen } \\
\text { las mismas oportunidades que una } \\
\text { persona joven para conseguir trabajo? }\end{array}$ & $\mathrm{x}$ & $\mathrm{x}$ \\
\hline $\begin{array}{l}\text { ¿Cuándo la persona adulta mayor se } \\
\text { enferma quién le auxilia? (ayuda)? }\end{array}$ & $\mathrm{x}$ & $\mathrm{x}$ \\
\hline $\begin{array}{l}\text { ¿Considera usted que aislar y } \\
\text { menospreciar a los adultos es normal en } \\
\text { la sociedad? }\end{array}$ & & $\mathrm{x}$ \\
\hline $\begin{array}{l}\text { ¿Sigo trabajando igual como en años } \\
\text { anteriores? }\end{array}$ & & $\mathrm{x}$ \\
\hline $\begin{array}{l}\text { ¿Necesito de algún familiar o amistades } \\
\text { para realizar actividades en el día, no } \\
\text { puedo hacerlo solo? }\end{array}$ & & $\mathrm{x}$ \\
\hline $\begin{array}{l}\text { ¿Me siento querido e importante para } \\
\text { mi familia y mi comunidad? }\end{array}$ & & $\mathrm{x}$ \\
\hline $\begin{array}{l}\text { ¿Participo en las decisiones de mi } \\
\text { barrio/comunidad? }\end{array}$ & & $\mathrm{x}$ \\
\hline $\begin{array}{l}\text { ¿Mi familia me trata con respeto y } \\
\text { cariño y cuida de mi bienestar? }\end{array}$ & & $\mathrm{x}$ \\
\hline $\begin{array}{l}\text { ¿Me siento abandonado/aislado por mi } \\
\text { familia? }\end{array}$ & & $\mathrm{x}$ \\
\hline $\begin{array}{l}\text { ¿Algún familiar se ha apropiado de mis } \\
\text { bienes o dinero sin mi consentimiento? }\end{array}$ & & $\mathrm{x}$ \\
\hline $\begin{array}{l}\text { ¿Mi familia me obliga a realizar } \\
\text { actividades en casa, para ganarme la } \\
\text { comida diaria? }\end{array}$ & & $\mathrm{x}$ \\
\hline $\begin{array}{l}\text { ¿Conozco las leyes que protegen a los } \\
\text { adultos mayores? }\end{array}$ & & $\mathrm{x}$ \\
\hline $\begin{array}{l}\text { ¿Los adultos mayores tenemos las } \\
\text { mismas oportunidades que los jóvenes } \\
\text { para conseguir trabajo? }\end{array}$ & & $\mathrm{x}$ \\
\hline $\begin{array}{l}\text { ¿Las personas piensan que los adultos } \\
\text { mayores somos inútiles? }\end{array}$ & & $\mathrm{x}$ \\
\hline $\begin{array}{l}\text { ¿Me siento discriminado por las } \\
\text { personas debido a mi edad? }\end{array}$ & & $\mathrm{x}$ \\
\hline $\begin{array}{l}\text { ¿Cuándo me siento enfermo tengo una } \\
\text { atención de salud inmediata? }\end{array}$ & & $\mathrm{x}$ \\
\hline ¿Qué mensaje dejaría para los jóvenes? & & $\mathrm{x}$ \\
\hline
\end{tabular}

*El nombre del encuestado no se solicitó por confidencialidad. 
Tabla 2.

Articulación de la encuesta, entrevista a profundidad, operacionalización de la variable violencia simbólica.

\begin{tabular}{|c|c|c|c|}
\hline Variable & Categoría & Unidades semánticas & Instrumento \\
\hline \multirow[t]{4}{*}{$\begin{array}{l}\text { Violencia } \\
\text { Simbólica }\end{array}$} & 1.- Autopercepción & $\begin{array}{l}\text { Envejecimiento, } \\
\text { salud-enfermedad, } \\
\text { etnia, } \\
\text { estado de ánimo }\end{array}$ & $\begin{array}{l}\text { Encuesta de } \\
\text { percepción de } \\
\text { la violencia } \\
\text { simbólica }\end{array}$ \\
\hline & $\begin{array}{l}\text { 2.- Contexto } \\
\text { familiar }\end{array}$ & $\begin{array}{l}\text { Indiferencia o } \\
\text { menosprecio } \\
\text { Agresión } \\
\text { Abandono } \\
\text { Independencia } \\
\text { Dependencia }\end{array}$ & \\
\hline & 3.- Contexto social & \multicolumn{2}{|c|}{$\begin{array}{l}\text { Discriminación por la etnia } \\
\text { Discriminación por edad } \\
\text { Reconocimiento de la comunidad }\end{array}$} \\
\hline & $\begin{array}{l}\text { 4.-Apoyo y } \\
\text { asistencia social } \\
\text { del Estado }\end{array}$ & \multicolumn{2}{|c|}{$\begin{array}{l}\text { Calidad de atención en servicios de } \\
\text { asistencia } \\
\text { Recursos institucionales, Apoyo } \\
\text { gubernamental }\end{array}$} \\
\hline
\end{tabular}

\section{Criterios de inclusión y exclusión}

Para la recolección de información, en el primer momento se consideró a las personas voluntarias que asistieron en calidad de participantes a la invitación a la casa barrial Pucahuaico, las cuales debían encontrarse en el rango de edad de 18 años en adelante, con responsabilidad frente a la población adulta mayor, ya fueran familiares cercanos y/o moradores del sector, directos o indirectos en la convivencia con esta población, cuyas características respondieran hacia una identificación étnica, en la que, sostenido a través de la entrevista, pueda ser entendido con reciprocidad de la pregunta y la respuesta, bajo el principio de voluntariedad, que permita identificar a la población adulta mayor con mayores condiciones de riesgo y vulnerabilidad.

El criterio de exclusión tomó como base principal todos aquellos parámetros contrarios a los criterios de inclusión que se justifican por alguna de estas razones: adolescentes menores de 17 años de edad, por requerir autorización escrita de un adulto para su entrevista; moradores del sector, ausentes en este proceso. Elementos considerados en el presente estudio (ver tabla 3 ). 
Tabla 3:

Criterios a considerar en la recolección de información.

Criterios de inclusión

Asistentes a la reunión convocada en Pucahuaico, independiente de su identificación étnica (adultos mayores desde los 65 años en adelante)

Participación voluntaria a la presente investigación mediante la firma del consentimiento informado.

Encuesta preliminar: Rango de edad de 18 a 85 años. Encuesta a profundidad: Rango de edad de 65 en adelante.

\section{Criterios de exclusión:}

Problemas sensoriales asociados al envejecimiento normal

\section{Principales hallazgos y discusión}

Los resultados se exponen sobre la base de los datos obtenidos en las encuestas, mediante un análisis descriptivo. Para la información obtenida en las entrevistas se utilizó un análisis cualitativo.

\section{Descriptivo de las encuestas}

Contexto general: las encuestas se realizaron a 46 personas de las cuales $59 \%$ fueron mujeres y $41 \%$ hombres. De esta población, 29 personas son mayores de 65 años (14 mujeres y 15 hombres). En cuanto al nivel de educación de la población encuestada, no se registró personas con nivel de educación técnica o superior, de ellas, una persona tiene educación secundaria, el 52\% educación primaria, mientras que el $46 \%$ señala no haber ido a la escuela en ningún grado de escolaridad. Analizando la encuesta por categorías se puede describir los siguientes principales hallazgos:

Referente a la categoría autopercepción, el 39\% de la población se autoidentifica como mestiza. De las personas que se identifican como indígenas (61\%), el 63\% son mujeres, mientras que en los hombres es el $48 \%$; no obstante, en las entrevistas se observa que las personas que se autoidentifican como mestizas, en su mayoría reflejan en su vestimenta la cultura indígena.

Referente a la categoría contexto familiar podemos observar que respecto de la percepción de la población menor de 65 años acerca de la situación laboral/económica de los adultos mayores, el 100\% de los encuestados considera que los adultos mayores no reciben remuneración alguna por los trabajos que realizan. 
Otro de los ítems referentes a la categoría "trabajo" manifiesta que, de los adultos mayores, el $90 \%$ no puede trabajar como en años anteriores; además, existe un $24 \%$ de adultos mayores que expresa no sentirse querido ni importante para su familia, mientras que un $29 \%$ manifiesta la ausencia de respeto y cuidado y de su bienestar. A estos datos podemos añadir que, de este grupo poblacional, el $47 \%$ necesita ayuda para movilizarse, volviéndose dependientes de un familiar para poder lograrlo.

En la categoría social se obtuvo que el $52 \%$ de los adultos mayores entrevistados mencionan que no participan en la toma de decisiones de la comunidad y que el $41 \%$ lo hace a veces (no son tomados en cuenta o la comunidad no logra generar interés en la participación), mientras que en la categoría asistencia social del Estado se pudo determinar que, de los servicios de atención médica, la mayoría mencionan que no existe o no recibe (88\%), y el $65 \%$ no conoce las leyes y derechos de servicio médico que los protegen.

Mediante un cruce de variables descriptivas entre algunas unidades semánticas de la categoría "contexto familiar" con la categoría "contexto social" se pudo determinar que el 79\% de los adultos mayores se considera discriminado debido a su edad (55\% si se considera discriminado siempre y $24 \%$ discriminado frecuentemente). El abandono familiar también tiene un valor importante: el $58 \%$ se considera de alguna manera abandonado por parte de su familia ( $24 \%$, si se considera abandonado y $34 \%$ abandonado de manera ocasional).

\section{Análisis de las entrevistas}

El análisis de las entrevistas a los adultos mayores de Pucahuaico se realizó bajo la óptica de cuatro categorías: autopercepción, contexto familiar, contexto social, apoyo y asistencia social del Estado.

\section{Autopercepción}

La categoría "autopercepción" contiene las unidades temáticas etnia, envejecimiento, estado de ánimo y salud enfermedad.

La sistematización de las entrevistas nos indica que la mayoría no habla ni entiende el Kichwa, no utiliza los atuendos indígenas porque, como un entrevistado afirmó, "No usa vestimenta porque no es indí- 
gena". Algunos expresan que antes los usaban o utilizaban atuendos puntuales, como poncho o sombrero, pero no el conjunto completo. A lo mencionado se suma la presión social, una entrevistada mencionó: "Verá, la ropita mía era de indígena, mi esposo me hizo cambiar".

Sobre envejecimiento y salud enfermedad, la mayoría reconoce la presencia de enfermedades y el no poder trabajar como principales características de malestar; además de no tener familia que la cuide de manera permanente. Un entrevistado afirma que: "Una desventaja es que hay personas a las cuales las personas o los hijos los hacen a un lado". En el mismo sentido, un adulto mayor señaló: "Toca ir uno mismo, más antes nos sabían vender en las boticas, decían que traiga la receta para vender, ahora si ya venden cualquier cosa... no hay nadie, tenemos que ir nosotros". La mayoría no se acompaña de nadie para buscar atención médica y reconocen el dolor físico y el cansancio como síntomas crónicos.

En relación con el estado de ánimo de los adultos mayores, las entrevistas indican que es bajo, acompañado de sentimientos de cansancio y tranquila resignación frente a las condiciones propias del envejecimiento, sus consecuencias y sus relaciones familiares. Una entrevistada mencionó sobre su estado de ánimo que: "Uhhh... yo no quiero seguir envejeciendo porque por la enfermedad es que yo estoy así... ¿no ve que me flaqueo y me engordo? Tengo, ya le digo, ese decaimiento por la enfermedad, son pocas las veces que amanezco con ánimos". Otro entrevistado, refiriéndose a su energía para enfrentar el día a día, expresa: "Es que ya no se tiene ánimos de trabajar; o sea, mientras uno es más joven, se tiene ánimo de caminar rápido, hacer las cosas rápido, en cambio ya, parece que con la edad ya va disminuyendo las fuerzas".

\section{Contexto familiar}

Las unidades temáticas de la categoría "contexto familiar" son: abandono, agresión, independencia-dependencia e indiferencia o menosprecio.

Sobre la experiencia de abandono, más de la mitad reitera su sentir de abandono total o parcial por parte de sus familiares. Al relatar su experiencia, un adulto mayor, manifiesta: "Porque, así como tengo un hermano en Quitoy tiene hijos, pero no tienen la posibilidad de venir 
a verme y visitarme". Otro adulto mayor menciona: "Aquí así, de la soledad, así es lo que vivimos... Eso de llevarme quiere mi hija, a vivir allá en la Loma y yo no quiero porque tengo aquí una casita viejita, trabajada con mi difunta mujer, ¿cómo la he de abandonar? Porque abandonando se desbarata la casita, tengo que utilizar "pis", eso". En muchos casos, parte de las razones del distanciamiento, voluntario o no, entre los adultos mayores y sus familias, también ronda alrededor de las diferencias en los estilos de vida, el lugar de la vivienda o trabajo de las nuevas generaciones, y el aferrarse de los mayores a sus propiedades y actividades agrícolas. Una entrevistada cuenta su experiencia con las siguientes palabras: "A veces... ¡ay, vieja molestosa!, cuando uno se les llama jay, está molestando la vieja! Han de decir, ya para qué será entonces, o sea, a veces ya parece que les estorbamos, como que somos molestosos, estamos preguntando que cómo están"

El análisis de dependencia-independencia refleja que seis de cada 10 adultos mayores dependen de su propio trabajo y se atienden a sí mismos; un ejemplo es lo señalado por uno de los entrevistados, que manifiesta: "Yo mismo vendo lo que cosecho para comprando mis cositas. Yo de repente le doy unos $\$ 20$ a la hija para que compre alguna cosita. Ni mis hijas me dan ni yo les doy". Por otro lado, tres de 10 personas dependen económicamente del bono de desarrollo humano, de sus hijos o de su pareja, como lo afirma una entrevistada al describir su experiencia: "Ah sí, de repente mis hijitos me ayudan, de ahí yo no cobro el bono ni nada, no nos ayudan si vea ellos que son de la tercera edad no ayudan, de repente hago tortillitas me voy al vóley a vender allá, pero más por distraerme, por ver como juegan mis hijos".

Sobre indiferencia y menosprecio, tres de cada 10 adultos mayores entrevistados se sienten menospreciados por sus familias, un ejemplo de esto es el testimonio de una entrevistada cuando dice: "Sí, porque las primas ni se acuerdan, ni las sobrinas de mi marido; viven cerca unas se llevan, otras no, pero no vienen a vernos. Sí, me han tratado mal, una prima es casada con uno que anda robando, le había robado a mi tía una vaquita, nos han hecho sufrir y mejor nos hemos retirado, pero tenemos al juez Dios". Asimismo, dos de cada 10 adultos mayores entrevistados manifiestan que no son visitados por sus hijos, como uno de ellos indica: "Como le digo, por lo que no nos vienen a ver a mis 
hijas les digo, claro han de esperar que me muera, de ahí han de venir a ver, eso es lo único que les digo directamente, de ahí nada más". Por otro lado, cuatro de cada 10 entrevistados no se siente menospreciado, se siente respetado y recibe visitas de sus hijos.

\section{Contexto social}

La categoría "contexto social" incluye las unidades semánticas de discriminación por edad, discriminación por etnia y reconocimiento de la comunidad.

De los participantes, siete de cada 10 señalan que los adultos mayores sufren discriminación por su edad, que son criticados y que la fuente de discriminación son los propios vecinos. Tres de cada 10 indican que no son discriminados y que se los invita a participar de las actividades de la comunidad. Un testimonio sobresaliente sobre este tipo de discriminación es el expresado por una participante: “A veces sí, sí me he sentido menospreciada por los jóvenes. Es que los jóvenes a veces se burlan de una... Si les quedan viendo, los ven con una indiferencia... había unos jóvenes que me trataban mal y un día ya me cansé, nos gritaban muerta, a mi esposo de muerto, nos insultaban... yo me subí al curso y les dije: a ver quién es el que está insultando, nadie habló, cogí el camino y me di la vuelta cuando yo estaba saliendo, ya para seguir para allá y seguir caminando sale un tipo y comienza a llamarme vieja ruca ni sé qué...."

$\mathrm{Al}$ igual que en la unidad anterior, siete de cada 10 describen que hay una gran discriminación por su etnia, como lo respalda el testimonio de una entrevistada, acerca de la situación de una menor, familiar suyo, en la escuela de la localidad: "Ella sufrió este menosprecio por parte de los compañeros de la escuela, ya que la molestaban diciendo que era india, hedionda y que todavía sufre desprecio de la demás gente por vestir de esa forma (atuendo indígena)". Otro testimonio, en un contexto diferente, señala la presión social y la discriminación no solo por parte de los mestizos, sino también por parte de otras minorías étnicas: "si cuando trabajaba mi esposo en la línea, trabajaba por adentro en el tren, entonces verá, ahí había los morenos ellos nos decían 'puendas', porque íbamos con el traje, como era muy caliente allá ahí, ya me cambié para ponernos solo blusita, porque ellos nos decían estas puendas. Ahí decía mi marido que se 
burlan de mí y por eso me saqué. Cuando el ponernos nuestro traje es un orgullo".

Sobre el reconocimiento de la comunidad, esta unidad semántica muestra una posición claramente definida, en la cual la mitad expresa sentirse excluido y no participar, mientras que la otra mitad expresa sentirse valorado y participar de las actividades de la comunidad. Hay un grupo menor que manifiesta que no conoce a la gente de Pucahuaico, o que participa solo de los eventos y fiestas organizados por el gobierno local.

El siguiente testimonio refleja estos datos: "Para cualquier cosa sí toman en cuenta, si no toman en cuenta porque somos mayores y que necesitamos así, hay algo de gente como es la reina del turismo hacen grupos y nos dieron para Navidad así cualquier cosa".

\section{Apoyo y asistencia social del Estado}

La categoría "apoyo y asistencia social del Estado" se compone de las unidades semánticas apoyo gubernamental, calidad de atención en servicios de asistencia y recursos institucionales.

Sobre el apoyo gubernamental, las entrevistas reflejan el deseo de la comunidad de que el apoyo del Estado se exprese con el bono de desarrollo humano y atención en salud principalmente (seis de cada 10). Una participante menciona: "Cuando estaba Correa de presidente, nos llevó de paseo, no recuerdo, pero era una parte de Esmeraldas, a todos los mayores; nos daban víveres y se acabó, Ahora dice que no hay plata y no hay nada. Ahora dicen y no sabe cómo vivimos y no saben si comemos o no comemos. Apoyar lo sé, porque ya no podemos trabajar, ya no tenemos... ya digo, estábamos muy contentos de que siquiera teníamos lo que nos estaban dando, el bono... y nos quitaron y ya no tenemos nada, nada".

En relación con la calidad de atención en servicios de asistencia en la comunidad, la mayoría afirma su acceso a servicios básicos y servicios en salud; sin embargo, reportan las demoras en la atención médica, la falta de medicinas y la deficiencia en alcantarillado como las principales limitaciones; como manifiesta una entrevistada: "Sí, sí tenemos. Sino que mi casa está al filo de la quebrada y nos da miedo que rebote y que se derrumbe y está en una parte peligrosa, y como está 
en una quebrada y como está en una pendiente baja, porque se vive la parte peligrosa, sabes que no lo bajan del cerro absorbiendo todo lo que sé, lo menos ya sabe que estamos en un pendiente en la casa viene una tubería porque pasa por ahí corre el agua de ahí".

Finalmente, sobre la atención con recursos institucionales de la comunidad, las respuestas indican la falta de acciones o presencia del Estado, mientras que los gobiernos locales tienen mejor connotación en la opinión de los encuestados. Un testimonio al respecto de un participante indica: "El presidente de la comuna sí se preocupa porque no había alcantarillado hace tiempo, porque nadie tenía, y entró un presidente y por él pusieron el alcantarillado a todos... El municipio no, el municipio no hace nada".

\section{Conclusiones}

En la vida cotidiana de los adultos mayores de Pucahuaico se pudo establecer varios parámetros que dan muestra de la condición de doble vulnerabilidad en el contexto diario de los adultos mayores. Si bien al inicio de la investigación hubo resistencia a proporcionar información, el trabajo con grupos focales, el acercamiento a sus líderes, a los adultos mayores de la comunidad, con técnicas de participación y empoderamiento, entrevistas y visitas domiciliarias, generó empatía, acceso, afecto y respeto recíproco en la comunidad, tanto en el grupo entrevistado, como en los/las adultos y jóvenes de la comunidad.

La investigación realizada da cuenta de la condición de vida de la población adulta mayor de Pucahuaico, en la que es visible la extrema pobreza en la que viven, la soledad, el abandono, la carencia de alimentos y de vivienda, el aislamiento, el silencio de sus voces, la dependencia de los familiares que los cuidan o el abandono por haber envejecido solos. A esto se suma la ausencia de afiliación a un seguro médico privado o a un seguro público del Estado. En consecuencia, el deterioro de su salud, incipiente atención médica y/o tratamiento de sus dolencias o enfermedades asociadas a determinantes sociales que han incidido en la vulneración de sus derechos a una vida digna.

En Pucahuaico la gran mayoría de los adultos mayores $(79 \%)$, se siente rechazado por su propia familia y comunidad debido a su edad; al igual, sienten que han perdido el afecto y cariño familiar. Otro foco 
de malestar viene del abandono del Estado en cuanto a su salud (el $88 \%$ no recibe atención sanitaria), así como la ausencia de exigencias en la atención médica, sobre todo por el desconocimiento de sus derechos. Existe un abandono casi total (93\%) en relación con la toma de decisiones colectivas, pues mencionan que no son tomados en cuenta o tienen una participación muy esporádica en estas decisiones.

Los resultados de las entrevistas nos indican que, en la comunidad de Pucahuaico, un porcentaje elevado de los adultos mayores enfrentan enfermedades degenerativas frecuentes, que se agravan por el limitado acompañamiento que reciben de sus familias, carencia de acceso a los servicios de salud y de recursos económicos para el suministro de medicinas. Estos tres determinantes sociales - vejez, enfermedad y abandono- fomentan un decaimiento en sus capacidades de afrontamiento emocional, expresado en desánimo, depresión y soledad. De la misma forma, en el contexto social señalan que hay una discriminación basada en su etnia y en su edad, aunque reconocen que la comunidad les invita a participar de las actividades organizadas por el gobierno local; en relación con sus familias, muchos manifiestan como problemático el abandono total o parcial por parte de los hijos y/o sus familiares, además de explotación laboral en actividades de cuidado no remuneradas. Por otra parte, se evidencia que hay un proceso de aculturación basado en la idea peyorativa de ser indígena, lo que se expresa en la presión de los miembros de las familias para dejar atuendos, lenguaje e idiosincrasia indígena. Paradójicamente, hay un impulso proveniente del gobierno local y de otros colectivos para recuperar la identidad cultural de los pueblos originarios. En este marco simbólico de convivencia de los adultos mayores se configuran ciertas ceremonias, que dan soporte a sus vivencias entre la solidaridad desarrollada entre pares que atraviesa por los cuerpos a través de la oralidad; es decir, de la palabra: "aquí vivimos y hemos de morir, hay amigos viejos como yo" "vivieron nuestros hijos", "aquí vivieron mi papá, mi mamá", "había el río, éramos fuertes", "yo tumbaba una vaca"; otros, "todo el día sembraba", "hacíamos los choclos para la minga", "el maíz, la papa no faltaba", "ahora ya no hay nada, ..."; "yo estoy encerrado en mi cuarto", "no saben de pronto me puedo morir y no saben nada", "en el cuarto que vivo, vive cerca un hijo, ellos me convidan el bocado"; "no tengo 
trabajo y eso es lo malo, hay mayores que todavía pueden trabajar en cualquier cosa"; "solo Dios sabe hasta cuándo me va a tener"; "verá, la ropita mía era de indígena, mi esposo me hizo cambiar"; "pongo solo el anaco de Otavalo"; "no me toman en cuenta en mi barrio"; "es normal que no nos miren"; "hacerse viejito es sufrir".

Diálogos que se vuelven una práctica de reflexión y enseñanza, guardada en la memoria, a través de sus formas de vida, saberes y aquella solidaridad que los identifica entre "iguales", porque aprendieron a convivir y envejecer juntos, a mirarse en las diferencias, contrario a la mirada del "otro" que individualiza y discrimina. A decir de Carballeda (2017), citado en Hermida y Meschini (2018), estas formas de violencia han subordinado e invisibilizado al otro. En nuestro caso, una violencia invisible que se naturaliza en la vida cotidiana de los adultos mayores.

El Trabajo Social tiene un reto en el empoderamiento de la población a través de la exigibilidad social que parte de la autonomía, la solidaridad y la autodeterminación para hacer visible la violencia simbólica que se incrusta en el abandono, olvido y naturalización de la condiciones de vida de la población adulta mayor de Pucahuaico, visible en la permanencia a la intemperie de algunos adultos mayores, mientras llegan sus familiares para abrir sus viviendas, en aquellos que requieren de asistencia para movilizarse de su habitación y la "solidaridad entre pares" que permite que se visiten unos a otros y conocer cómo se encuentran; y/o aquellos que sus dolencias por enfermedad los han abandonado en el silencio y su deseo de muerte antes que la vida.

Es fundamental que los saberes, la solidaridad y la memoria histórica de la población adulta mayor recobre la importancia histórica en los niños, jóvenes y adultos de la comunidad, priorizando la interacción en las relaciones sociales en el discurso, la acción y la palabra, en la toma de decisiones comunitaria como un derecho y no como un beneficio social.

Los contenidos curriculares de educación básica, secundaria pregrado y posgrado deben incluir en sus mallas el abordaje de la población adulta mayor, sensibilización y prevención de acciones en política pública hacia el futuro. Elementos que cobran vida desde un Trabajo 
Social específico en el tema de envejecimiento y la calidad de vida de los adultos mayores. Un Trabajo Social que, para Cazzaniga (2000), debe ser "situado" en problematizar y socializar las condiciones de los seres humanos, que si bien el discurso de los derechos humanos se ha imbricado en la política social, este "discurso" es visible en similar situación en las condiciones de la población adulta mayor de Pucahuaico, ausente de mecanismos efectivos de cumplimiento de sus derechos humanos y perspectiva de género, que les permita desarrollar su propio proyecto de vida.

En este tránsito de ir y venir de los adultos mayores, la intervención del Trabajo Social es desde el respeto a sus saberes, sus tradiciones y rechazando el colonialismo impuesto. Porque de acuerdo con el pensamiento de Hermida y Meschini (2018), no se trata de pugnar por tiempos pasados, sino de encontrarnos en la resistencia a la valoración de nuestras raíces, generar motivación al conocimiento e identidad por parte de los jóvenes y adultos de Pucahuaico. Se trata, entonces, retomando a Cazzaniga (2000), de empoderamiento colectivo, en el que el término "beneficiarios" se ha desplazado de acciones no sostenibles a largo plazo, y que contrariamente objetivan a los seres humanos. El desafío está en las garantías efectivas de cumplimiento como "sujetos de derechos", que dignifiquen la vida de una población que crece demográficamente y, con ella el abandono, el olvido por las instituciones estatales de protección y por la sociedad.

Finalmente, es responsabilidad del Estado y sus instituciones dar respuestas efectivas en la tutela de derechos, así como la corresponsabilidad de la sociedad y la academia, intervenir en la complejidad social para hacer de un problema privado un problema público; una acción encaminada a la restitución de un derecho humano desde la construcción de sus propias realidades y necesidades como concepto básico del "vivir bien", recuperando la identidad de nuestros adultos mayores y las tradiciones; fortaleciendo la solidaridad; recuperando la minga como fiesta del reencuentro y las reuniones del Cabildo basadas en la participación colectiva, para la toma de decisiones comunitarias que deconstruyan aquellas prácticas impuestas y nos construyan con la sabiduría de los pueblos.

Reflexión que ha surgido de la investigación propuesta: realizar un Trabajo Social decolonial, respetuoso de los saberes y tradiciones co- 
munitarias, que se inserta en la vida de las comunidades revalorizando sus capacidades y potencialidades, devolviendo a ellos los resultados para su propia autodeterminación y construcción colectiva de estrategias que revaloricen a su población adulta mayor y, con ella, mejores condiciones de vida, rompiendo barreras, visibilizando la cultura y la herencia histórica comunitaria.

\section{Bibliografía}

Agüero, J. (2018). El mundo de la vida en Trabajo Social. La comprensión de los sujetos sociales y sus mundos de vida para una intervención social significativa y emancipadora en Trabajo Social. Ciudad Autónoma de Buenos Aires: Prometeo Libros.

Alvarado García, A. M. y Salazar Maya, Á. María. (2014). Análisis del concepto de envejecimiento. Gerokomos, 25(2), 57-62. DOI: https://dx.doi.org/10.4321/S1134-928X2014000200002

Bourdieu, P. (1991). Language and symbolic power. Harvard University Press.

Bourdieu, P. (2008). El sentido práctico. España: Siglo XXI Editores.

Bourdieu, P. (1999). La miseria del mundo (Vol. 1). Ediciones Ákal.

Carballeda, A. (2017). La negación del Otro como violencia. Pensamiento decolonial y cuestión social. En P. Meschini y M. E. Hermida (Comp.), Trabajo Social y Descolonialidad. Epistemologías insurgentes para la intervención en lo social. Mar del Plata: Editorial de la Universidad Nacional de Mar del Plata.

Casanova Moreno, M. de la C., Trasancos Delgado, M., Corvea Collazo, Y., Pérez Sierra, M. y Prats Álvarez, O. M. (2013). Manifestaciones de violencia intrafamiliar hacia adultos mayores diabéticos. Pinar del Rio, 2012. Revista Cubana de Medicina General Integral, 29(2):160-172.

Cortez, R., Muñoz Martínez, R. y Ponce-Jiménez, P. (2020). Vulnerabilidad estructural de los pueblos indígenas ante el COVID-19. Boletín sobre covid-19, 7-8. Recuperado de https://www.researchgate.net/publication/343403302_Vulnerabilidad_estructural_de_los_pueblos_indigenas_ante_el_COVID-19

Cazzaniga, S. (2020). Acerca del otro (otra, otre) en trabajo social. Concepciones y problematizaciones. Debate Público - Reflexión de Trabajo Social, 10(19), 47-54. 
Yepes Delgado, F. L. y Hernández Enríquez, C. (2010). Haciendo visible lo invisible: violencia de género y entre generaciones en una comunidad indígena colombiana. Investigación y educación en enfermería, 28(3): 444-453.

Espín-León, A., Jimeno-Morenilla, A., Pertegal-Felices, M. \& AzorínLópez. J. (2021). Effect of Migration Flows on the Ethnic Identity of the Indigenous Peoples of the Amazonian Communities. Springer International Publishing.

Francés, M. Á. (2005). Socialización, educación y reproducción cultural: Bordieu y Bernstein. Revista interuniversitaria de formación del profesorado, 19(1), 159-174.

Feito, L. (2007). Vulnerabilidad. Anales del sistema sanitario de Nava$r r a, 30,07-22$. Fernández, J. M. F. (2005). La noción de violencia simbólica en la obra de Pierre Bourdieu: Una aproximación crítica. Cuadernos de trabajo social, (18), 7-31.

GADP San Antonio de Ibarra. (2015). Plan de Desarrollo y Ordenamiento Territorial

GADP San Antonio de Ibarra. (s. f.). San Antonio de Ibarra Pueblo Mágico. Recuperado 8 de marzo de 2021 de https://gadsanantonioibarra.gob.ec/

Galtung, J. (1969). Violence, peace, and peace research. Journal of Peace Research, 6(3), 167-191.

Gómez Ricárdez, L. A., Rodríguez Abrego, G. y Krug Llamas, E. (2007). Prevalencia y factores asociados a violencia familiar en adultos mayores de Ocozocoautla (Chiapas, México). Revista Española de Geriatría y Gerontología, 42(1): 27-34. DOI: 10.1016/S0211-139X(07)73518-7.

Guedes, D. T., Curcio, C. L., Alvarado Llano, B., Zunzunegui, M. V. y Guerra, R. (2015). SciELO - Salud Pública - None None. Revista Panamericana de Salud Pública 37: 293-300.

Herrera, D. y Terán, S. (2011). Análisis del valor que representa para los pobladores la identidad cultural de la parroquia de San Antonio de Ibarra y propuesta alternativa. (Tesis de Grado). Universidad Técnica del Norte.

Hermida, M. E. y Meschini, P. A. (2018). Trabajo social y descolonialidad: epistemologías en lo insurgente para la intervención en lo social. Interacción y perspectiva: Revista de Trabajo Social, $8(2), 215-215$. 
Hermida, M. E. (2018). Habitar las instituciones: notas para una intervención social-otra en contextos de colonialidad. Ponencia presentada en II Jornadas Internas del Instituto de Estudios Críticos en Humanidades, Universidad Nacional de Rosario, Argentina.

Morales Garzón, E. A. (2017). Costumbre y tradiciones de los grupos étnicos de Imbabura. (Tesis de Grado). Pontificia Universidad Católica del Ecuador Sede Ibarra

Miranda, L., Aguirre, A. y Espín, A. (2020). Percepciones sobre discriminación y formas de violencia en los/las estudiantes de la Universidad Central del Ecuador (2018). Revista Ciencias Sociales, 1(42).

Ospina, E. L. S. y Jiménez, C. E. (2018). Vulnerabilidad social de la población desterrada. Más allá del goce efectivo de derechos. Revista de Ciencias Sociales, 24(4), 38-56.

Pillemer, K. \& Moore, D. W. (1990). Highlights from a Study of Abuse of Patients in Nursing Homes. Journal of Elder Abuse \& Neglect, 2(1-2): 5-30. DOI: 10.1300/J084v02n01_02.

Rodríguez Calvo, M. D., Gómez Mendoza, C., Guevara de León, T., Arribas Llopis, A., Duarte Durán, Y. y Ruiz Álvarez, P. (2018). Violencia intrafamiliar en el adulto mayor. Revista Archivo Médico de Camagüey, 22(2): 204-213.

Muñoz Loayza, B. A. (2018) Ventajas y desventajas del muestreo probabilístico y no probabilístico en investigaciones científicas. (Examen Complexivo). Ecuador, Machala: Universidad Técnica de Machala.

Otzen, T. y Manterola, C. (2017). Técnicas de Muestreo sobre una Población a Estudio. International journal of morphology, 35(1), 227-232.

Ungar, M. (2008). Resilience across Cultures, The British Journal of Social Work, 38(2), 218-235. DOI: https://doi.org/10.1093/ bjsw/bcl343

Pizarro Hofer, R. (2001). La vulnerabilidad social y sus desafíos: una mirada desde América Latina. Cepal.

Villa, M. y Rivadeneira, L. (2000). El proceso de envejecimiento de la población de América Latina y el Caribe: una expresión de la transición demográfica. Encuentro Latinoamericano y Caribeño sobre las Personas de Edad. Santiago de Chile: División 
de Población-Centro Latinoamericano y Caribeño de Demografía, CELADE.

Viteri, B., Villalva, O. y César, M. (1988). Monografía de San Antonio de Ibarra. Documento auspiciado por el Consejo Provincial de Imbabura, el artista Gilberto Almeida E. y editado por el IADAP. 